\title{
Glomerular Basement Membrane Spikes Present
}

National Cancer Institute

\section{Source}

National Cancer Institute. Glomerular Basement Membrane Spikes Present. NCI

Thesaurus. Code C83473.

A finding indicating the presence of protrusions or extensions in the basement

membrane of the glomeruli. 